\title{
Interactive comment on "Impact of temperature dependence on the possible contribution of organics to new particle formation in the atmosphere" by Fangqun Yu et al.
}

\section{Anonymous Referee \#2}

Received and published: 16 December 2016

The authors implement a temperature-dependent organic nucleation parameterisation in a global model. Simulations are compared with in situ observations. A climate forcing is calculated. The modification of the parameterisation and the in-situ observation comparison are interesting and useful, but the climate forcing calculation is questionable.

The main problem is that (looking at Yu et al. 2015), there seems to ONLY be organicsmediated nucleation in this model set-up. This does not invalidate all of the findings, but one of the main outcomes of the CERN CLOUD experiment is that we know there are many different types of nucleation going on at the same time, and so models which only include one type of nucleation will seem more sensitive to changes than the real 
atmosphere. Figure 5 shows that the temperature dependent parameterisation does give very good agreement with observations, and I believe the temperature dependence is useful and valid in this sense. But the idea that $40 \%$ of boundary layer CCN would be lost to a temperature dependence in the organic nucleation rate only makes sense if no other nucleation is happening in the model, which is very unrealistic for the atmosphere. This also calls into question the quoted radiative forcings (radiative effects, since they do not relate to the pre-industrial?) shown in Figure 6.

Simulations which include other nucleation types should be performed to give a more accurate estimate of the climate estimates, which I am sure will be smaller than those quoted in the current version of the manuscript.

Was there any physical basis for setting the maximum value of fT to 10 ? If the method is valid for higher temperatures, why is it not valid for lower temperatures? If the method gives unrealistic values at low temperatures, why do we trust it at high ones?

It says in Section 2.2 that this is the "first global modeling attempt in studying the effect of temperature on organics-mediated nucleation in the atmosphere", and this assertion is repeated in the summary and discussion section. Section 20 of supplementary materials and figure S9 in Dunne et al. (2016)'s Nature paper on nucleation based on CERN CLOUD experiment contains a temperature dependence of organic nucleation. It was not the main focus of the paper but it should be mentioned.

With revisions, the paper will make a good contribution to the field and should be published. But the emphasis should be placed on the realistic improvements in nucleation representation shown in Figure 5, rather than on unrealistically high model sensitivity to an overly simplistic nucleation scheme. 